\title{
Role of Nasal Endoscopy in Sinonasal Diseases
}

\author{
${ }^{1}$ Ritesh Shelkar, ${ }^{2}$ Jeevan Vedi, ${ }^{3}$ Seema Patel, ${ }^{4} \mathrm{KS}$ Dasgupta, ${ }^{5}$ Kanchan Lanjewar
}

\begin{abstract}
Aims and objectives: To evaluate sinunasal diseases with the help of nasal endoscopy. To study efficacy of nasal endoscopy in diagnosing nasal pathology over clinical examination. To define medical and surgical functional endoscopic sinus surgery (FESS), management according to type of nasal pathology. To define applications of nasal endoscopy (biopsy, swab, epistaxis control, foreign body removal, rhinolith removal, follow-up).
\end{abstract}

Materials and methods: Total 100 patients were studied. Patients came with complaints of nasal blocking, nasal discharge, mass in nasal cavity, bleeding etc, included in study. Pre-endoscopic assessment was carried out like history, examination, investigation. Endoscopic was done after consent under necessary anesthesia. Endoscopy was done using 0 and $30^{\circ}$ endoscope with three standard passes.

Result: Total 100 patients were studied. Male to female ratio was 1.8:1. Out of 100 patients maximum number of patients had chronic sinusitis on nasal endoscopy examination (22); followed by nasal polyp (27) and deviated nasal septum and epistaxis (10). Nasal endoscopy was an excellent diagnostic aid in condition like epistaxis, nasal mass, nasal obstruction, foreign body, nasopharyngeal tumor.

Conclusion: Diagnostic nasal endoscopy offers high diagnostic accuracy in patient with sinonasal complaints. Diagnostic nasal endoscopy is gold standard tool in patient having sinonasal complaints. It has high accuracy due to vision control, has less bleeding, minimal complication, and early postoperative recovery. It is a good tool for diagnosing anatomical variation.

Keywords: Anatomical variation, Chronic sinusitis, Nasal endoscopy, Nasal passes.

How to cite this article: Shelkar R, Vedi J, Patel S, Dasgupta KS, Lanjewar K. Role of Nasal Endoscopy in Sinonasal Diseases. Clin Rhinol An Int J 2015;8(1):8-11.

Source of support: Nil

Conflict of interest: None

\section{INTRODUCTION}

In 1901, Hirschmann ${ }^{1}$ first used the modified cystoscope to examine middle meatus. Based on the experience and

\footnotetext{
${ }^{1,5}$ Assistant Professor, ${ }^{2}$ Professor, ${ }^{3}$ Associate Professor ${ }^{4}$ Professor and Head

${ }^{1-5}$ Department of ENT, Indira Gandhi Government Medical College, Nagpur, Maharashtra, India
}

Corresponding Author: Ritesh Shelkar, Assistant Professor Department of ENT, \#230 Medical Road, Opposite Ambedkar High School, Untakhana, Nagpur-440009, Maharashtra, India e-mail: riteshnshelkar@gmail.com teaching of Messerklinger, Stammberger and Kennedy, ${ }^{2-4}$ the diagnosis and treatment of inflammatory sinus disease continue to evolved. Nasal endoscopy allows detailed and complete evaluation of intranasal anatomy and identification of pathology, that is impossible to see using standard techniques with headlight or head mirror. With the endoscope, the surgeon gains capacity for precise anatomy identification and angled, illuminated, magnified viewing of the internal nose preoperative, intraoperatively, and postoperatively. As an added benefit, an attached camera can provide a photographic demonstration to the patient or create documentation for the permanent record. ${ }^{5}$ Recently combination of diagnostic endoscopy and imaging study has become the corner stone in the evaluation of the paranasal sinus diseases. This is the basis of the new concept of the functional endoscopic sinus surgery (FESS).

The following study was undertaken in order to ascertain the efficacy of endoscopy in diagnosing a spectrum of nasal and nasopharyngeal pathology, which otherwise remain unrevealed clinically.

\section{AIMS AND OBJECTIVES}

- To evaluate sinonasal diseases with the help of nasal endoscopy.

- To study efficacy of nasal endoscopy in diagnosing nasal pathology over clinical examination.

- To define medical and surgical (FESS) management according to type of nasal pathology.

- To define applications of nasal endoscopy (biopsy, swab, epistaxis control, foreign body removal, rhinolith removal, follow-up).

\section{MATERIALS AND METHODS}

It was a prospective study. The study was conducted at Indira Gandhi Government Medical College during a period from August 2009 to December 2013. Total 100 patients were studied. Inclusion criteria were patient presenting with nasal complaints like nasal blockage, running nose, bleeding from nose, nasal mass, foul breath, foreign body in nose, and patient above 10 years of age. Exclusion criteria were patient with acute infection of nose and paranasal sinuses, and age less than 10 years. Local ethics committee approval was acquired for this study. 
A detailed history and ENT examination was done. Written and informed consent was taken before the diagnostic nasal endoscopy. Zero degree, $30^{\circ}$ rigid nasal endoscope were used $(4 \mathrm{~mm})$. All diagnostic nasal endoscopies were performed under local or general anesthesia. Nasal cavity was packed with patty of $4 \%$ xylocaine with adrenaline (1:1000) or xylomethazoline/ oxymethazoline. A complete examination was successfully accomplished in an organized manner with three mentioned nasal passes of the endoscopy. The findings of nasal endoscopy were recorded in the proforma. Various endoscopic assisted procedures and surgeries were done as and if required. Patients were followed-up after medical or surgical management at intervals of 1 week, 1 month, 3 months, and 6 months.

\section{OBSERVATION AND RESULT}

Total 100 patients were studied. The age ranged from 11 years to 80 years. Maximum patients were in 31 to 40 years of age group, which contribute $26 \%$ of total patients. In study male, preponderance was $65 \%$ and female was $35 \%$, Male to Female ratio was 1.8:1.

In study most common complaint was nasal discharge seen in 32 patients (32\%), followed by nasal obstruction in $24(24 \%)$, while least common complaints was foreign body in nose $3(3 \%)$. Many patients came with multiple complaints at a time for particular pathology, most common symptom with, which patients presented considered as a primary complaints (Table 1).

Most common finding on anterior rhinoscopy was nasal discharge seen in 46 patients, followed by deviated nasal septum, nasal polyp and inferior turbinate hypertrophy seen in 21 patients. Least common finding was synechia in one patient. Most common finding on nasal endoscopy was middle meatus discharge seen in 40 patients, followed by polyp in 30 patients, followed by inferior turbinate hypertrophy in 26 cases.

Most common anatomical variation seen in nasal endoscopy was spur it was seen in 10 patients, followed by concha bullosa in seven patients. Anatomical variation was most commonly associated with chronic sinusitis; out

Table 1: Presenting primary complaints of patients

\begin{tabular}{lll}
\hline Symptoms & No. of patients & Percentage \\
\hline Nasal discharge & 32 & 32 \\
Nasal obstruction & 24 & 24 \\
Nasal bleeding & 15 & 15 \\
Nasal mass & 15 & 15 \\
Foul breath & 6 & 6 \\
Olfactory disturbance & 5 & 5 \\
Foreign body & 3 & 3 \\
\hline
\end{tabular}

of 22 patients of chronic sinusitis anatomical variation was seen in 16 patients $(72.72 \%$ ) (Table 2). Patients were grouped on basis of presenting chief primary complaints and studied.

\section{Patients with Nasal Discharge}

Thirty-two patients had primary complaint as nasal discharge. Out of 32 patients, eight patients had ethmoidal polyposis, 15 patients of chronic sinusitis, Antrochoanal polyp seen in three patients, two patients had deviated nasal septum, allergic rhinitis in three patients. One patient on endoscopy had clear watery fluid discharge from frontal resses. Computed tomography showed defect in cribriform plate and final diagnosis of cerebral spinal fluid (CSF) rhinorrhea was confirmed.

\section{Patients with Nasal Obstruction}

Twenty-four patients had primary complaint of nasal obstruction. Out of 24, eight patients had deviated nasal septum. Two patients were of maxillary malignancy, the biopsy was taken from the mass endoscopically. Three patients of nasal obstruction had ethmoidal polyposis on nasal endoscopy, which was not seen on anterior rhinoscopy. Four patients had chronic sinusitis. Antrochoanal polyp was seen in three patients and three had inverted papilloma, patients with inverted papilloma showed polypoidal mass on anterior rhinoscopy, nasal endoscopy was done and biopsy was taken for histopathological examination. One patient had synechia in right nostril with history of traumatic epistaxis in past, routine anterior rhinoscopy examination not showed any synechia. These patient underwent endoscopic release of synechia.

\section{Patients with Nasal Bleeding}

Fifteen patients presented as nasal bleed. Six patients had epistaxis in woodruffs area; in these patients anterior rhinoscopy examination was normal. These patients were managed by endoscopic cauterization. Four patients had bleeding and congestion in Little's area on anterior rhinoscopy. Nasal endoscopy was done to find out other bleeding site and status of nasal cavity. These patients were also managed by endoscopic cauterization. Two

Table 2: Anatomical variation

\begin{tabular}{ll}
\hline Anatomical variants on nasal endoscopy & No. of patients \\
\hline Concha bullosa & 7 \\
Bulla ethmoidlis & 4 \\
Paradoxical turbinate & 6 \\
Accessory ostea & 1 \\
Spur & 10 \\
Total & 28 \\
\hline
\end{tabular}


patients of nasal angiofibroma presented with history of nasal bleed. Nasal endoscopy was done to locate the size, extension of mass and site of bleeding in operation theatre only with preparation for general anesthesia if needed, nasal endoscopy was done as non-touch technique to avoid epistaxis. Rest three patients showed mass in nasal cavity. Endoscopic biopsy was done and histopathological examination report suggestive of squamous cell carcinoma, hemangioma and rhinosporidiosis in each.

\section{Patients with Olfactory Disturbances}

Five patients had olfactory disturbance. All these patients were case of atrophic rhinitis. Out of these five, one patient had history of Hansen's disease; other two had history of nasal myasis. Diagnostic nasal endoscopy revealed exact pathology; crust was present in all of them. On nasal endoscopy of these, two patients had bony and one show cartilage perforation over septum. Atrophic rhinitis patients were managed by counseling, nasal douches and regular endoscopic removal of crust.

\section{Patients with Nasal Mass}

Fifteen patients with nasal mass were included in study. Four patients were of ethmoidal polyposis, six had antrochoanal polyp, while chronic sinusitis feature was seen in two patients. In two patients with history of nasal mass endoscopic biopsy was taken and histopathological examination report was suggestive of hemangioma and rhinosporidisis respectively. One patient, who presented with nasal mass had nasal angiofibroma.

\section{Patients with Foul Breath}

Six patients present with such complaint. One patient had normal clinical finding, and show foreign body in right nasal cavity on endoscopy, and was removed with the help of endoscope. One had chronic sinusitis, while four patients of foul breath had atrophic rhinitis.

\section{Patients with Foreign Body Nose}

Three patients presented with foreign body in nose. In two patients, there was mucoid discharge in right nasal cavity and one had normal clinical finding. It was only by nasal endoscopy, that foreign body was identified and removed. In one patient, foreign body was present posteriorly in nasal cavity.

In this study, out of 100 patients, 39 patients had no pathology on routine clinical examination related to particular diseases, which was further confirmed after doing nasal endoscopy.

Out of 100 patients, maximum number of patients had chronic sinusitis on nasal endoscopy examination (22); followed by nasal polyp (27) and deviated nasal septum and epistaxis (10) (Table 3).

\section{DISCUSSION}

The development of modern rigid nasal endoscopy represents a major advance in rhinologic diagnostic capability. The study conducted by Bakari et $\mathrm{al}^{6}$ and Levine et $\mathrm{al}^{7}$ had maximum number of patients in between 31 to 40 years with mean age 33.3 and 35.6 respectively. In our study, majority of patients was in the age group of 31 to 40 years with total 26 cases (mean 39.1). In the present study, 65 patients were male while 35 patients were female with male to female ratio was 1.8:1. In the study conducted by Kirtane et $\mathrm{al}^{8}$ there were 48 (61.5\%) males and $30(38.4 \%)$ females and male to female ratio was 1.6:1. Tabaee ${ }^{9}$ had 39 (63.9\%) male and 22(36\%) female with ratio 1.7:1 in his study. Similarly, study conducted by Bakari et $\mathrm{al}^{6}$ showed 42 (55.2\%) male and 34 (44.7\%) female and had ratio 1.2:1. In the study conducted by Kirtane et $\mathrm{al}^{8}$ the commonest complaint was nasal discharge seen in $61 \%$ patients, followed by nasal obstruction in 59\% patients. In the study, conducted by Bakari et $\mathrm{al}^{6}$ the nasal discharge (97.4\%) was the most common presenting complaints followed by nasal obstruction ( $94.7 \%)$. Out of 22 patients of sinusitis, 16 (72.72\%) patients had associated anatomical variations on diagnostic nasal endoscopy. This was well in agreement with the study done by Lloyd et al ${ }^{10}$ who reported a figure of $62 \%$. Similarly, study conducted by Levine et $\mathrm{al}^{7}$ showed anatomical variation in $56.6 \%$ in his 150 studied patients.

Diagnostic nasal endoscopy was of great significant in patients of epistaxis. It helped in accurate diagnosis of cause of epistaxis and proper management of the same. This measure was better tolerated and less uncomfortable

Table 3: Diagnosis of patient on nasal endoscopy

\begin{tabular}{ll}
\hline Diagnosis & No. of patients \\
\hline Chronic sinusitis & 22 \\
Ethmoidal polyposis & 15 \\
Antrochoanal polyp & 12 \\
Deviated nasal septum & 10 \\
Epistaxis & 10 \\
Atrophic rhinitis & 9 \\
Rhinolith & 4 \\
Carcinoma maxilla & 3 \\
Inverted papilloma & 3 \\
Angiofibroma & 3 \\
Allergic rhinitis & 3 \\
Hemangioma & 2 \\
Rhinosporidisis & 2 \\
CSF rhinorrhea & 1 \\
Nasal synechia & 1 \\
\hline Total & 100 \\
\hline
\end{tabular}


as compared to nasal pack or balloon. This conclusion was consistent with those of McGarry. ${ }^{11}$

Diagnostic nasal endoscopy was useful in identifying conductive olfactory loss and associated pathology with it. Clinical examination failed to diagnose pathology in three out of five $(60 \%)$ cases of olfactory loss and endoscopy was necessary to smake the proper diagnosis. This figure is close to the figure of $51 \%$ given by Allen et al. ${ }^{12}$

Rigid endoscopy helped in careful manipulation and removal of nasal foreign bodies and rhinolith under direct vision, which were posteriorly placed and were not visible on clinical examination. Also, posterior extent of rhinolith was carefully evaluated. This conclusion was also supported by studies of Keck et $\mathrm{al}^{13}$ and Hade et al. ${ }^{14}$ Nasal endoscopy helps in exact localization and minimizing trauma to surrounding structure and prevents bleeding during foreign body removal.

In this study endoscopic biopsy was taken in six patients with sinonasal mass. Nasal endoscopy showed exact site in the region of pathology from where biopsy had to be taken which help in accurate histopathological diagnosis and help to minimize the bias. This conclusion was supported in the study conducted by Tabaee et $\mathrm{al}^{9}$ who stated that office based nasal endoscopy with biopsy represent a safe and important tool in evaluation of sinonasal neoplasm and this procedure provides diagnostic information that may alter treatment decision.

In this study, five patient of olfactory disturbance had atrophic rhinitis, which was best diagnosed and managed by nasal endoscopy. This conclusion was supported by studies of Yuca et $\mathrm{al}^{15}$ who managed cases of atrophic rhinitis on regular follow-up and endoscopic removal of nasal crust. In our study, anterior rhinoscopy did not reveal pathology and diagnosis in 39 cases (39\%) which were diagnosed on nasal endoscopy. This finding is consistent with Levine et $\mathrm{al}^{7}$ study showed a figure of $38.7 \%$. Thus, nasal endoscopy is efficient over clinical examination for diagnosing nasal and nasopharyngeal pathologies.

\section{CONCLUSION}

The worldwide acceptance of nasal endoscopes as an important diagnostic tool and useful surgical aid has contributed much to the world of Rhinology. It allows an unparalleled vision with brilliant illumination of nose and paranasal sinuses. In our study, we see that nasal endoscopy was an excellent diagnostic aid in many situations like sinusitis, unexplained headache, epistaxis, olfactory disturbances, nasal masses, nasal polyposis, nasal obstruction, nasal foreign bodies, nasal discharge, sinonasal malignancies. Diagnostic nasal endoscopy offers a high diagnostic accuracy in patients with sinonasal complaints. Diagnostic nasal endoscopy is capable of detecting nasal and nasopharyngeal pathologies, which would otherwise be missed and this supports diagnostic nasal endoscopy as investigation of gold standard in the field of rhinology. Some nasal fossa pathologies are better defined on diagnostic nasal endoscopy. Endoscopic directed procedures have high accuracy due to vision controlled and incomparable guidance in treatment of nasal and nasopharyngeal pathologies. Clinical examination and diagnostic nasal endoscopy are complementary in making correct diagnosis. Diagnostic endoscopy must be done prior to any functional endoscopic sinus surgery, as they help in assessing the extent of sinus diseases and to know the variation and vital relation of the paranasal sinuses.

\section{REFERENCES}

1. Rice DH, Schaefer SD. Endoscopic paranasal sinus surgery. 3rd ed. 2004. p. 15.

2. Messerklinger W. Endoscopy of the nose. Urban and Schwarzenberg; 1978.

3. Stammberger H. Functional endoscopic sinus surgery. BC Decker 1991;102.

4. Kennedy DW. Prognostic factors, outcomes and staging in ethmoid sinus surgery. Laryngoscope 1992;102:1-18.

5. Brown's S. Otorhinolaryngology, head and neck surgery. Rodney J Schlosser, David W Kennedy. Nasal Endoscopy. 7th ed. 2008;2:1344.

6. Bakari A, Afolabi OA, Adoga AA, et al. Clinico pathological profile of sinonasal masses. Biomedical Central Research Notes 2010;3:186.

7. Levine HL. The office diagnosis of nasal and sinus disorders using rigid nasal endoscopy. Otolaryngol Head Neck Surg 1990;370-373.

8. Kirtane MV. Functional endoscopic sinus surgery: a preliminary study. Ind J Otolaryn 1991;43:126-129.

9. Tabaee A, Hsu AK, Kicker A. Indications, technique, safety, and accuracy of office-based nasal endoscopy with biopsy for sinonasal neoplasm. Int Forum of Allergy and Rhinology 2011;1:225-228.

10. Lloyd GAS, Lund VJ, Scadding GK. Computerized tomography in the preoperative evaluation of functional endoscopic sinus surgery. J Laryngol Otol 1991;105:181-185.

11. McGarry GW. Nasal endoscope in posterior epistaxis: a preliminary evaluation. J Laryngol Otol 1991;105:428-431.

12. Seiden AM, Duncan HJ. The diagnosis of a conductive olfactory loss. Laryngoscope 2001;111:9-14.

13. Keck T, Liener K, Strater J, et al. Rhinolith of the nasal septum. Int J Pedi Otorhinolaryngol 2000;53:225-228.

14. Hade U, Ghossaini S, Zaytoun G. Rhinolithiasis: a forgotten entity. Otolaryngol Head and Neck Surg 2002;126:48-51.

15. Yuca SA, Yuca KB, Yakut F, et al. A forgotten difficult entity: ozena report of two cases. East J Med 2010;15:114. 